\title{
On the Practicality of Group Dynamic Assessment: A Seminal Enterprise Deserving Closer Scrutiny
}

\author{
Andisheh Saniei (Corresponding author) \\ ELT Department, Science and Research Branch, Islamic Azad University, Tehran, Iran \\ E-mail: andisheh_saniei@yahoo.com \\ Parviz Birjandi \\ ELT Department, Science and Research Branch, Islamic Azad University, Tehran, Iran \\ E-mail: pbirjand@yahoo.com \\ Esmaeel Abdollahzadeh \\ ELT Department, Iran University of Science and Technology, Tehran, Iran \\ E-mail: s_abdollah@iust.ac.ir
}

Received: 12-07-2014

Accepted: 22-09-2014

Published: 01-03-2015

doi:10.7575/aiac.ijalel.v.4n.2p.39

URL: http://dx.doi.org/10.7575/aiac.ijalel.v.4n.2p.39

\begin{abstract}
This paper is a preliminary study designed to scrutinize the way Group Dynamic Assessment (G-DA), suggested by Poehner (2009), has been implemented in L2 classrooms regarding its basic premise: moving the group forward in its ZPD while benefiting individuals as well. Since the one-to-one interaction that characterizes DA framework represents an unrealistic model for classroom teachers who must manage classes of 15 to 30 learners, Poehner (2009) suggests the use of DA with groups of L2 learners rather than individuals and offers examples of two approaches to G-DA: concurrent and cumulative. However, the development of group ZPD cannot be easily traced in the presented approaches, and the process of shifting the activity from the individual to the group is not clarified either. To present a more realistic model of G-DA, this paper suggests the application of small group DA to L2 classrooms. To this end, it has initially delved into the concepts of 'group learning', 'group ZPD', and 'group DA'. Next, the areas of ambiguity in the two suggested approaches to G-DA have been identified. Finally, a less complicated model of classroom DA, namely small group DA is presented.
\end{abstract}

Keywords: group learning, group ZPD, G-DA, small group DA, pair DA

\section{Background and Purpose}

Dynamic Assessment has recently received attention from second language learning researchers and practitioners since it offers a conceptual framework which integrates assessment into instruction and posits that learners' responsiveness to instruction can be seen as a measure of learners' potential. The outstanding feature of this mode of assessment is teachers intervening whenever needed to help learners perform beyond their current level of capabilities. DA derives from Vygotsky's (1988) belief that abilities are not innate but emergent and dynamic. Echoing Vygotsky, Lantolf and Poehner (2004) argue that learners' cognitive abilities are in the state of flux and can change through mediation. In fact, it is the mediator's assistance that would lead to the learner's cognitive development at the potential level, and the difference between learner's actual (unassisted) level and potential (assisted) level is known as the zone of proximal development (ZPD) ( Lantolf, 2006; Ohta, 2000).

Although the theoretical construct of ZPD essentially focuses on the communication between novice learners and more capable others, the scope of ZPD encompasses more than novice-expert interactions. In other words, people working together are able to co-construct contexts in which expertise emerges as a feature of the group (Lantolf, 2006). Therefore, a major challenge facing the existing literature is how to use DA in a social context, such as L2 classroom, where the teacher should interact with not just a single ZPD but a group of ZPDs. This possibly led Poehner (2009) to develop a new framework, group dynamic assessment, in which it is possible for the mediator to negotiate with a group of learners to co-construct several ZPDs while moving the entire group forward in its ZPD. He also argues that dyadic and group-based DA follows the same principle of co-constructing a ZPD; however, they differ in that G-DA must take into account the group's ZPD as well. He then offers two different approaches to G-DA (concurrent and cumulative) and provides examples for the implementation of each in L2 classrooms.

Undoubtedly, Poehner's innovative notion of G-DA proves attractive because it offers to L2 teachers and practitioners a more realistic model of applying DA with groups of learners rather than individuals; however, the applicability of the suggested approaches to G-DA triggers drawbacks to this economical mode of DA. This study aims at addressing the inadequacies of the two approaches to G-DA (concurrent and cumulative) by Poehner (2009). It also intends to uncover the real function of G-DA, i.e. promoting both group's and individuals' ZPDs. Finally, appreciating the innovative 
notion of G-DA, this paper suggests small group DA as a more realistic and less complicated model of group DA for classroom teachers.

\section{Group and group learning}

In recent decades, the concept of group learning in the classroom context has preoccupied L2 researchers and practitioners by questioning what constitutes a group and how group learning can take place. In Petrovsky's (1983) terms, group is a collective whose main characteristic is the joint activity among its members. Similarly, group work is a joint activity whose purpose is the sharing of knowledge and abilities that would lead to the development of both the group and the individuals. In the same vein, Donato (1994) argues that it is through the collective that individuals can learn how to solve their problems, the property that is absent in loosely-knit group since its members do not work together toward a common goal.

Perhaps the meaning of group can best be clarified by Leont'ev's Activity Theory which makes sense of individual actions within a broader, collaborative setting. Leont'ev (1981) suggested the notion of activity with the example of hunting among hunter-gathered people, in which individuals can engage in a collective activity stimulated by common needs or goals. In other words, it is the motive (the need) and goal of activity that determines the level of group functioning in a socio-cultural context. In Vygotskian perspective, as Donato (1988) notes, what is implicit at the core of group activity is not the interaction of individuals as independent of one another, but rather, how they work together to construct a shared context in which learning occurs. This is in line with the formation of what Poehner (2009) calls group ZPD following a number of individual ZPDs working together in a joint activity.

To clarify the meaning of group in his study, Poehner (2009) elaborates on Petrovsky's (1985) explanation of three major approaches to relating individual and group abilities and calls them 'group-as-context', 'group -as-cooperation', and 'group -as-collective' models respectively. The meaning of group in the first model, according to Petrovsky (1985), is limited to a total of interrelated individuals who interact to achieve a predetermined aim for which understanding individual cognitive processes is unimportant. On this view, the group itself is regarded as a contextual variable that ignores individuals' abilities.

In the second model, group-as-cooperation, the individuals' abilities and goals are appreciated while their interrelations with other group members' goals have also been emphasized. And finally, the third model is defined by Petrovsky (1983, p. 14) as "a number of individuals whose interactions and interrelations are realized through the aims, tasks, and values of common activity". When the groups adopt a collective orientation, the goal of the joint activity shifts from the individual to the group to cause the recovery of every group member since "the health of each is the wealth of all" (Petrovsky, 1985, p. 161).

Regarding the concept of joint activity, Poehner (2009) defines the group as a psychological entity composed of individuals with different forms of expertise working together to perform a task that no single individual was able to accomplish independently. This view, which originates from Petrovsky's definition of group as 'collective', stipulates the leading role of teachers in group works as engaging learners in activities that are challenging to all and mediating prompts, at different levels and quantities, which can benefit all group members. This way, the development of the group and the individuals are so interrelated that every mediating move should be directed to the entire group.

\section{Group Dynamic Assessment}

After establishing the meaning of group and group learning, the next issue needing more clarification is the question of how to assess the group and its ZPD. While Vygotsky's original formulation of the ZPD was particularly concerned with the interaction between 'novice' and 'expert', current sociocultural theorists, as Mitchell, Myles, and Marsden ( 2013) point out, "have expanded the concept to include pair and group work among peers"(p.241). This was earlier emphasized by Lantolf and Pavlenko (1995) who argued that "individuals, none of whom qualifies as an expert, can often come together in a collaborative posture and jointly construct a ZPD in which each person contributes something to, and takes something away from, the interaction" (p. 116). Similarly, Donato (1994) asserts that learners are able to provide guided support to their peers during collaborative interaction in ways almost similar to expert scaffolding documented in the existing literature. In fact, peer scaffolding enables the learners to expand their own L2 knowledge as well as extending the knowledge of their peers. The idea of peer, or better to say collective, scaffolding suggests that in case of encountering a problem, the contributions from each individual can provide supportive scaffolds for other members and help the group to solve the problem collectively. In other words, what the group can do together stands higher than what the individuals can do alone; i.e., the group ZPD is larger than the individual ZPD.

On the other hand, Poehner (2009) contends that the one-to-one format of DA which characterizes most DA and ZPD research to date has become a challenge to classroom teachers who interact with a group of ZPDs. In the same vein, Saniei (2012) investigated that EFL teachers in Iran mostly believe that applying DA in classroom contexts where more than twenty students participate would be time- and energy-consuming and seem less feasible compared with nondynamic assessment. Accordingly, the essence of interaction within the microcosm of class was modified by Poehner (2009) based on a new framework known as group dynamic assessment in which it is possible for the mediator to simultaneously negotiate with a group of learners to co-construct several ZPDs. This way, the collaboration of the individuals is believed to move the entire group forward in their ZPD as well (Lantolf and Poehner, 2008; Lantolf and Poehner, 2011; Poehner, 2009).

As a leading advocate of G-DA, Poehner (2009) claims that one-to-one (dyadic) and group-based DA follow the same principle of offering learners mediation to help them co-construct a ZPD; however, they differ in that group ZPD should 
also be considered in G-DA. As a case in point, Lantolf and Poehner (2010) report the results of a G-DA study in which the teacher does not run through the full range of mediating prompts with a single learner before beginning again with another individual. The teacher's focus, instead, remains fixed on the entire class while calling on a particular student to answer a question and then addressing another to continue the contribution. This way, the teacher is claimed to move the entire class forward in its ZPD through co-constructing ZPDs with individuals. Similarly, the role of teacher is highly emphasized by Poehner (2009) in promoting the development of all group members through actively engaging the entire group in G-DA interaction. In this regard, Poehner (2009) distinguishes 'primary' from 'secondary' interactants during G-DA. The primary interactants include the teacher and one of the students who receive his/her mediation and the secondary interactants are other students who listen and benefit from the teacher-student exchanges. For example, the teacher may pose a question to the entire group to which individual learners (primary interactants) will respond. However, the exchange occurs in the social space of the class and before the other group members to simultaneously benefit secondary interactants.

To display the implementation of G-DA in L2 classrooms, Poehner (2009) introduces two different approaches to GDA: concurrent and cumulative. In the concurrent approach, although the teacher provides mediation to a particular individual, the exchange initiated by the primary interactant in the form of question, struggle, or comment can provide the ground for another's contribution. For example, the teacher calls on a different student, other than the student who initiates the interaction, to reformulate the response given by the first student. In this way, the interaction shifts between primary and secondary interactants. In the second approach, cumulative G-DA, the students should take turn to engage as primary interactants with the teacher. When a student provides an incorrect answer, the teacher provides that same student with mediation prompts until s/he reaches the correct answer. This approach is believed to be cumulative since its goal is to move the group forward in its ZPD through negotiations with individual group members in their own ZPDs. "Cumulative G-DA attempts to move the group forward through co-constructing ZPDs with individuals, but concurrent G-DA supports the development of each individual by working within the group's ZPD"(Poehner, 2009, p. 488).

\section{The Ambiguities of G-DA}

An obvious advantage of interacting with groups of learners over a dyadic model of interaction is that mediating groups of learners instead of individuals is more realistic and less time- and energy-consuming to language teachers that must engage classes of over 20 learners. However, the notion of organizing teacher-student interaction in a whole class setting and using DA with groups of learners rather than individuals is a new enterprise that although seem familiar to most classroom practitioners, its application and management requires a more in-depth scrutiny. The central idea of GDA, as Poehner (2009) argues, is to enable teachers "to explore and promote the group's ZPD while also supporting the development of individual learners"(p. 471). This raises the question of how it is possible to manage group activities to reveal individual member's gradual development. In other words, with respect to the main concern of DA, discovering where learners encounter a problem and providing each with required support to solve them, the immediate question coming to mind is whether G-DA can identify individuals' areas of difficulties at different levels while engaging the whole class in an activity. To answer this potential question in the mind of classroom teachers and practitioners, Poehner (2009) points out teachers must actively engage the whole class, a large group, in G-DA interactions to promote the development of all group members. Engaging the entire group, he adds, does not mean that mediating individuals is ignored by the teacher, but that every mediation should be directed to the group as well. To this end, he distinguishes 'primary' from 'secondary' interactants in his study and claims that since the teacher's offered mediation in response to a given learner's difficulty and their negotiations as primary interactants occur before the other learners in the social space of the class, it will be beneficial to other group members who are secondary interactants.

What remains vague in Poehner's definition is whether the mediation given to learner A, a primary interactant, is virtually helpful for learner B, a secondary interactant, who may not have the same learner's difficulty to receive that same level of mediation. In other words, one critical issue in the context of group DA, as Davin and Donato (2013) note, is that only a particular number of students will be active participants who respond to teacher mediation and the rest will play the role of observant who are believed to benefit from primary interactants' negotiations. This may limit the cognitive engagement of a majority of secondary interactants in benefiting from the teacher's mediation (Davin, 2011). Another ambiguity in this regard is how individual class members can be given the equal chance of being involved in negotiation as primary interactants if there are only particular students who respond to teacher's questions and engage more overtly with the teacher. On the other hand, even if they are all given this chance, how it will be managed to save the class time (the real motive for innovating G-DA as a substitute for one-to-one interaction) is under question.

To provide evidence for concurrent G-DA, the first approach to G-DA, Poehner (2009) illustrates snapshots of two exchanges in Gibbon's (2003) study in which the class works in groups to conduct an experiment on magnetism. In this approach, the teacher does not wait for a single student to reformulate his/her response before beginning again with another student. Rather, the teacher indicates that the response is incorrect and provides mediating prompts, but then calls on a different student to reformulate the response. In this way, as Poehner (2009) claims, the teacher engages the entire class in the activity and offers mediation intended to benefit all the students. However, one obvious disadvantage of this approach, as Davin (2011) notes, is that since a student is not given the opportunity to reformulate her/his statement, s/he may face anxiety. Furthermore, concurrent DA may lower the confidence of a student who is not given the opportunity to offer a second response thus s/he never knows what the correct answer was on his/her own part. 
Compared with concurrent G-DA, cumulative G-DA in which students take turns engaging as primary interactants with the teacher seems more manageable. A good illustration of this presented by Poehner (2009) is the case of Tracy, a fulltime L2 Spanish teacher, who interacts with a class of students aged 9-10 years. Tracy stands in front of the class, where one of the students is also asked to go and perform on a task. Then, she intervenes to offer mediation when the student (primary interactant) experiences problems. Secondary interactants, the whole class, watch as Tracy is helping the primary interactant to solve a problem. When the primary interactant struggles to find an appropriate answer, his/her gaze shifts from the teacher to the class. This, according to Poehner (2009), means drawing the class into the interaction by looking at them. Once the interaction continues, involvement of other students become more evident, yet Tracy quiets them all. Even when secondary interactants raise their hands to contribute, Tracy does not call on them, rather she prefers to wait for the primary interactant to correctly respond - the evident dearth of collective activity. The second learner involved in the interaction with Tracy will be one of the volunteers who takes turn by raising his/her hand, and the story goes on with the rest of the individuals without displaying the sign of joint interaction. Although Tracy has successfully traced individuals' ZPD in her class through eight prefabricated mediating prompts ranging from most implicit to most explicit, the meaning of joint activity is missing from her work. In fact, what she does is not different from what other practitioners have done in dyadic models of DA, except for the performance of primary interactants at the front of the so-called beneficiary secondary interactants. Such performance is, albeit, absent in the previous DA works; nevertheless, it cannot show how and to what extent secondary interactants are benefitting from primary interactants to promote their own ZPDs. Furthermore, in the above-mentioned studies, there is no indication of group's ZPD development: the major drawback to both approaches to G-DA.

\section{How to manage G-DA through small group DA}

Based on the illustrated protocols, classroom DA seems only helpful for those students who are active participants in the interaction with their teacher as primary interactants. However, it may not be sufficient for monitoring the language development of every student in the classroom (Davin \& Donato, 2013) and does not seem capable of promoting all the students' ZPDs in a classroom context. Large group DA, as shown in Poehner's work, portrays little difference from the conventional teaching strategy that the majority of L2 teachers apply to interact with their students in a classroom setting. Undoubtedly, applying G-DA in a whole class as a large group is a different endeavor than standing before the class and mediating students who raise their hands, assuming this would benefit the rest as well. Whereas G-DA posits that the development of the group and that of the individuals are so interconnected that requires the individuals to carry out an activity cooperatively as they cannot do it independently, no sign of cooperation in its real sense can be traced in the presented snapshots. This may result from the fact that implementing G-DA in L2 classroom is an initiative that should be conducted in a step by step process. Perhaps a large group DA in which the entire class is assumed as a context for individual's participation is more challenging to L2 teachers than a small group DA. It may be more appropriate to divide the whole classroom first into small groups, including 2 to 3 members, and then to practice G-DA in small groups to reveal individual member's development as they participate cooperatively in a given activity. Within small groups, students often take responsibility for assisting each other and can learn through interaction with both their teacher and their peers; that is, they can benefit from both teacher- and peer-mediation (Davin \& Donato, 2013). Moreover, compared with large group DA, it is more feasible for L2 teachers to manage small groups and to consider each as a social entity in which two or three individuals share their knowledge and abilities to move the group forward in its ZPD.

Appreciating the innovative notion of G-DA as a more realistic and economical model of DA in L2 classrooms and in an attempt to present a more practical model for its application, the authors of this paper suggest small group DA, pair DA, founded on the same principle of G-DA; i.e. moving the group forward while benefiting individuals (Poehner, 2009). The suggested model somehow differs from the existing ones in that the teacher works within the ZPD of each pair in the classroom. This may imply that the development of pair ZPD is the offspring of both peer- and teachermediation; therefore, the type of mediation each pair receives must be distinguished in this regard. Identifying such a difference, of course, is not a challenging endeavor. The type and frequency of prompts each pair receives through DA process can be easily diagnosed through transcribing the audio-recorded interactions and distinguishing peer- from teacher-mediation.

Placing the students in small groups and assigning them to collaboratively complete a task will give all group members the opportunity to share their ideas and verbalize their thoughts while participating in a joint activity (Brooks \& Donato, 1994). Moreover, while large group DA provides only the active participants with the opportunity to receive teacher's mediation, small group DA offers all students both teacher's and peer's mediation. The following excerpts selected from the recorded interactions between an EFLteacher (one of the authors of this paper) and small group members in an Iranian EFL classroom illustrates how small group DA can be beneficial to both the primary and secondary interactants in small groups/pairs. In each protocol, M represents the mediator/teacher, PI stands for the primary interactant, and SI for the secondary interactant.

The teacher used DA framework during 9 fifty-to-sixty-minute sessions of task completion applied after the teacher's explicit instruction in rhetorical organization in writing in an EFL writing classroom. The students were twenty two freshmen of TEFL who took basic writing course and received DA enrichment program for nine weeks (sessions). The participants were divided into eleven pairs based on their performance on a researcher-made writing test. In the process of pairing, it was attempted to place each two learners with almost the same/similar writing ability in one pair. The rationale behind this way of grouping was preventing the establishment of novice-knower/less capable-more capable 
relationship between the 2 members of each pair so that pair ZPD could truly represent the interconnection between individual and group ZPDs.

Within the applied small group DA, the teacher responded to pairs' errors based on the taxonomy of implicit-to-explicit mediating prompts (see Table 1) while they were performing the given task. The purpose of offering the graduated prompts to each pair based on its level of responsiveness was measuring the distance between the actual and potential developmental levels (ZPD) of the pair, namely pair ZPD. The reduction in the number of prompts required by the pair, particularly reduction of explicit prompts, from session one to session nine was believed to indicate the pair ZPD's growth.

Table 1. Taxonomy of Implicit-to-Explicit Mediations

\begin{tabular}{ll}
\hline Level of Explicitness & Type of Mediation Move \\
\hline 1 & Accepting/Rejecting pair's response \\
\hline 2 & Reading the erroneous sentence with a questioning tone \\
\hline 3 & $\begin{array}{l}\text { Asking the pair to identify the problem (e.g., What is wrong with } \\
\text { this sentence?) }\end{array}$ \\
\hline 5 & $\begin{array}{l}\text { Identifying the place of error/problem and narrowing down its } \\
\text { location (e.g., repeating or pointing to the specific segment which } \\
\text { contains the error) }\end{array}$ \\
\hline 6 & $\begin{array}{l}\text { Indicating the nature of the error, but not identifying it directly (e.g., } \\
\text { There is something wrong with the used conjunction.) }\end{array}$ \\
\hline 7 & $\begin{array}{l}\text { Asking the learner to consider a possible solution (e.g., Write the } \\
\text { correct conjunction which shows contrast in your sentence.) }\end{array}$ \\
\hline 8 & $\begin{array}{l}\text { Offering a choice of possible solution (e.g., Conjunctions indicating } \\
\text { contrast are 'but' and 'yet'.) }\end{array}$ \\
\hline
\end{tabular}

As a case in point, consider the below excerpt in which the mediation was provided to help pair 11 remove the wrong verb 'result of' and write the correct verb 'result in' for the sentence 'For example, a stroke can result of nicotine in the blood stream'. The sentence is included in the paragraph written by the pair in response to task nine in which the learners were required to write a one-paragraph essay based on the given information. In order to make the process of data analysis easier, some of the sentences that were produced in Persian were translated into English while transcribing the audio-recorded interactions.

Protocol $A$

1. M: [Looking at both members of the pair] What do you mean by 'result of' in this sentence?

2. PI: We mean the result of nicotine in the blood stream is a stroke.

3. M: But 'result of' does not mean this here. You need to put a discourse marker here which plays the role of verb. 'Result of' is not a verb in English.

4. PI: Can we write 'result by'?

5. M: [Looking at both members of the pair] 'result by' is not a verb either. We have 'result in' and 'result from' as the verb in English, but not 'result of' or 'result by'.

6. PI: What about 'result in'?

7. SI: No...'result from'.

8. PI: Yeah, result from... result from nicotine

9. M: 'That's right'. Now can you tell me what 'result from' means?

10. PI: It is the result of... Stroke is the result of nicotine

11. SI: Nicotine causes stroke

12. M: Very good

In this protocol, the mediator's intervention began by checking whether or not the pair understands that the discourse marker 'result from' is replaced by the inappropriate verb 'result of' in their writing. After observing the pair's misunderstanding, the mediator explained the nature of the error to help the pair to consider another option (line 3). Following the wrong attempt of the pair (line 4), the teacher helped them again to consider another solution (line 5). This time both the primary interactant (PI) and the secondary interactant (SI) attempted to provide the correct answer (lines 6-8). In other words, the production of PI was corrected immediately by SI and then confirmed by PI as the solution to the problem. Moreover, the two members shift their role from PI to SI. That is to say, the member who once plays the role of primary interactant may have the role of secondary interactant for the second time. This contribution confirms the effect of G-DA interactions exchanged between the primary and the secondary interactants in a small group DA. Finally, to make sure the interaction has led to the complete understanding of the problem, the mediator 
asked for the meaning of the verb 'result from' as the pair offered the solution. PI begins defining the meaning (line 10) and SI helped him to complete it (line 11). This contribution can verify the role of each group member's involvement in a joint intellectual activity (Petrovsky, 1985).

Another illustration in which the collaboration of both pair members to come to correct answer can be revealed is the following excerpt taken from the interaction between P10 and the mediator. In this protocol, the pair was asked to add an appropriate discourse marker to the concluding sentence they had written in response to task eight (in session eight). The pair had written their conclusion as 'If you want to be healthy, the easiest way is using vitamins' without adding any concluding discourse marker at the beginning of the sentence.

Protocol B

1. M: Your conclusion lacks something.

2. P: [silent]

3. M: You have not used any discourse marker for drawing conclusion.

4. PI: So, if you want to be healthy, ...

5. M: Good. [looking at both pair members]You can also use other concluding markers, such as 'no wonder', 'obviuosly', 'as a result', or 'therefore'.

6. SI: Obviuosly... Obviously, if you want to be healthy, ....

7. M: Very good.

The episode reveals that indicating the nature of the error (line 1) and asking the pair to consider a possible solution (line 3) are not sufficient to elicit the appropriate response. Therefore, the teacher appeals to a more explicit mediation move and offers the possible solutions to the problem (line 5). The pair benefits from the provided mediation and selects the best option to resolve the problem. This protocol is a good illustration of how the mediator can invite both primary and secondary interactants to share their knowledge in order to move the small group forward in its ZPD while also benefiting individuals (Poehner, 2009). It also shows how the development of the group and the individuals are interrelated. In other words, the pair's ZPD development can be a good manifestation of either pair member's ZPD growth due to the fact that both members (who are believed to be at the same writing ability) share their ideas to solve a joint problem.

After confirming the fact that the development of the group and the individuals are interrelated, the next issue this paper deals with is how to measure pair's ZPD to represent individuals' ZPDs as well. From the SCT perspective, learners' progress in the ZPD can be tracked in terms of the type and the frequency of offered mediations (Poehner, 2005). Accordingly, fewer requests for prompts, as Aljaafreh and Lantolf (1994) note, imply less dependence on the mediator in performing a task, an observation that signifies a shift from other-regulation to self-regulation. Table 2 presents the frequency of meditational moves offered to a given pair (pair 11) in nine enrichment sessions.

Table 2. The Frequency of Meditational Moves Received by Pair11 for Performing Nine Tasks

\begin{tabular}{ll}
\hline Task Number & The Number of Received Mediational Moves \\
\hline One & 12 \\
\hline Two & 11 \\
\hline Three & 9 \\
\hline Four & 8 \\
\hline Five & 7 \\
\hline Six & 9 \\
\hline Seven & 7 \\
\hline Eight & 6 \\
\hline Nine & 5 \\
\hline
\end{tabular}

A comparison of the frequency of meditational strategies, displayed in the table, depicts a dramatic drop of the teacher's meditational moves for the pair 11 from task one to task nine. The reduced number of offered mediations, as mentioned before, indicates the extension of the pair's ZPD to higher levels. In other words, P11 who received 12 prompts for task one (as depicted by Table 2) required only 5 prompts for task 9 , an observation that confirms the pair's microgenetic growth across the mediation sessions. However, if we look at the pair's profile more carefully, we can see that the trend of the pair's ZPD growth is not necessarily a smooth, linear process (from task one to task nine):

\section{P11: $12 \begin{array}{llllllll}11 & 9 & 8 & 7 & 9 & 7 & 6 & 5\end{array}$}

It is clearly shown that there is a (more or less) downward trend for the number of mediations offered to Pair 11 as sessions progressed except for task six. The higher frequency of mediations for this task ( 9 mediations), compared with task five ( 7 mediations), can be related to the nature of task six which is more challenging than task five. This confirms Vygotsky's hypothesis that learners require more external mediations when faced with more challenging tasks. In other words, while the ZPD would predict an overall decrease in the amount of external mediation learners will require from session 1 to session 9, this does not mean that it will be a smooth, linear process (e.g., eight prompts during session 1, 
six prompts during session 2, five during session 3, etc.). Rather, we might expect backsliding (M. Poehner, Personal communication, 2014), whereby individuals require slightly more mediation during, for instance, session 6 than in session 5 (but still not as much as during session 1). In this way, there is overall forward movement but it is, as Vygotsky described it, a 'revolutionary' rather than 'evolutionary' process.

\section{Conclusion}

The notion of organizing teacher-student exchanges in a whole class setting in which individuals' abilities emerge as the outcome of joint activity is not innovative in nature. However, managing group activities while conducting dyadic interaction with group members, giving all individuals the chance of interacting with the teacher, and mediating them to promote both individual and group ZPD are not so practicable to L2 classrooms as Poehner (2009) claims. He argues that without a clear understanding of the relationship between individual development and that of the group, it is difficult to conceive of group DA. Therefore, he defines the group not as an assemblage of individuals, but as a psychological entity comprising individuals with different kinds of expertise who cooperatively carry out an activity that no single individual could do independently. This definition proves attractive because it seems to show the main purpose of joint activity. Yet it begins to appear vague when put into practice the way Poehner's illustrations of both approaches to G-DA display. In fact, none of the provided examples of G-DA approaches represent the true essence of cooperation; rather they lack the realistic manifestation of the relationship between individual's ZPD and that of the group.

The current literature reveals a strong need for more research focused on the implementation of DA in group settings (Saniei, 2014; Shabani, 2012). Although classroom DA is really helpful for teachers who intend to unite teaching and assessment in their classes, it might not be sufficient for the language development of all students due to time limitation (Davin \& Donato, 2013). With respect to the inadequacies of the two approaches to classroom DA, one area of investigation to address a more practical, realistic model of G-DA might involve assisting the students in small groups, i.e. pairs, as suggested by the present study. This way, the members in each pair are assisted by the mediator/teacher in case of having problem while performing a given task and are provided with the opportunity of receiving both peer- and teacher-mediation. However, the meaning of peer-mediation in this study does not reflect the interaction between knower and novice, or more capable or less capable, but between two capable learners in which no one is supposed to emerge as expert since expertise is a collective phenomenon (Brooks, Donato, \& Mcglone, 1997; Donato, 1994; Storch, 2002). This way of grouping can ensure that the small group's ZPD is the true manifestation of individual members' ZPDs who are at almost the same language learning ability and require the same level of assistance/ meditational move from their teacher who plays the role of knower or more capable other.

As a final remark, the major contribution of this paper might be to extend the typical dyadic format of DA to a small group model and to see its feasibility in an EFL writing classroom. Being concerned with limitations of one-to-one format of DA and favoring the innovative notion of G-DA as an appropriate substitute, the present study has been a small step towards uncovering the significant role of collaborative interaction in the ZPD progression and investigating the application of Vygotsky's concept of ZPD for the groups of learners rather than individual ones.

\section{References}

Aljaafreh, A., \& Lantolf, J. P. (1994). Negative feedback as regulation and second language learning in the zone of proximal development. The Modern Language Journal, 78, 465-483.

Brooks, F. B., \& Donato, R. (1994). Vygotskian approaches to understanding foreign language learner discourse during communicative tasks. Hispania, 77, 262-274.

Brooks, F. B., Donato, R., \& Mcglone, J. V. (1997). When are they going to say "it" right? Understanding learner talk during pair-work activity. Foreign Language Annals, 30, 524-541.

Davin, J. K. (2011). Group dynamic assessment in an early foreign language learning program: Tracking movement through the zone of proximal development. Unpublished Doctoral Dissertation, University of Pittsburgh, Pennsylvania.

Davin, K. J., \& Donato, R. (2013). Student collaboration and teacher-directed classroom dynamic assessment: A complementary pairing. Foreign Language Annals, 46 (1), 5-22.doi: 10.1111/flan.12012

Donato, R. (1988). Beyond group: A psycholinguistic rationale for collective activity in second language learning. Unpublished Doctoral Dissertation, University of Delvare, New York.

Donato, R. (1994). Collective scaffolding in second language learning. In J. P. Lantolf \& G. Apple (Eds.), Vygotskian approaches to second language learning research (pp. 33-56). Norwood, NJ: Albex.

Gibbons, P. (2003). Mediating language learning: Teacher interactions with ESL students in a content-based classroom. TESOL Quarterly, 37, 247-273.

Lantolf, J. P. (2006). Sociocultural theory and L2: State of the art. SSLA, 28,67-109.

Lantolf, J. P., \& Pavlenko, A. (1995). Sociocultural theory and second language acquisition. Annual Review of Applied Linguistics, 15, 108-124.

Lantolf, J. P., \& Poehner, M. E. (2004). Dynamic assessment in the language classroom. Pennsylvania: CALPER. 
Lantolf, J. P., \& Poehner, M. E. (2008). Sociocultural theory and the taching of second languages.London: Equinox Publishing.

Lantolf, J. P., \& Poehner, M. E. (2011). Dynamic assessment in the classroom: Vygotskian praxis for second language development. Language Teaching Research, 15, 323-340. doi: 10.1177/1362168810383328

Leont'ev, A. N. (1981). Problems of the development of the mind. Moscow: Progress.

Mitchell, R., Myles, F., \& Marsden, E. (2013). Second Language Learning Theories ( $3^{\text {rd }}$ ed.). London and New York: Routledge.

Ohta, A. S. (2000). Rethinking interaction in SLA: Developmentally appropriate assistance in the zone of proximal development and the acquisition of L2 grammar. In J. P. Lantolf (Ed.), Sociocultural theory and second language learning (pp. 51-78). Oxford: OUP.

Petrovsky, A. V. (1983). Towards the construction of a social psychology theory of the collective. Soviet Psychology, 21(2), 3-21.

Petrovsky, A. V. (1985). Studies in psychology. The collective and the individual. Moscow: Progress.

Poehner, M. E. (2009). Group dynamic assessment: Mediation for the L2 classroom. TESOL Quarterly, 43(3), 471-491.

Poehner, M. E. and Lantolf, J. P. (2005). Dynamic assessment in the language classroom. Language Teaching Research, 9 (3), 1-33.

Saniei, A. (2012). Dynamic assessment: A call for change in assessment. The Asian EFL Journal, 59 (4), 4-19.

Saniei, A. (2014). Raising Iranian EFL learners' pragmatic awareness of intercultural rhetoric in writing through group dynamic assessment (Unpublished doctoral dissertation). SRBIAU, Tehran, Iran.

Shabani, K. (2012). Group dynamic assessment: Instructional implications for L2 listening comprehension (Unpublished doctoral dissertation). University of Tehran, Tehran, Iran.

Storch, N. (2011). Collaborative writing in L2 contexts: Processes, outcomes, and future directions. Annual Review of Applied Linguistics, 31, 275-288.

Vygotsky, L.S. (1978). Mind in Society: The development of higher psychological processes. Cambridge, MA: Harvard University Press.

Vygotsky, L. S. (1986). Thought and language. Cambridge, MA: MIT Press. 